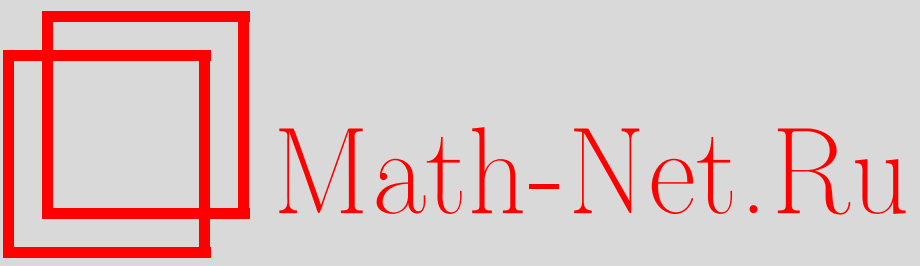

А. В. Одесский, В. Н. Рубцов, Полиномиальные алгебры Пуассона с регулярной структурой симплектических листов, ТМФ, 2002, том 133, номер 1, 3-23

DOI: https://doi.org/10.4213/tmf377

Использование Общероссийского математического портала Math-Net.Ru подразумевает, что вы прочитали и согласны с пользовательским соглашением

http://www.mathnet.ru/rus/agreement

Параметры загрузки:

IP : 18.209 .158 .208

26 апреля 2023 г., 13:00:34 
ТЕОРЕТИЧЕСКАЯ

И МАТЕМАТИЧЕСКАЯ

ФИЗИКА

Том 133, № 1

октябрь, 2002

(C) 2002 г.

А.В. Одесский* ${ }^{*}$ В.Н. Рубцов ${ }^{\dagger}$

\section{ПОЛИНОМИАЛЬНЫЕ АЛГЕБРЫ ПУАССОНА С РЕГУЛЯРНОЙ СТРУКТУРОЙ СИМПЛЕКТИЧЕСКИХ ЛИСТОВ}

Исследуются полиномиальные алгебры Пуассона с определенными условиями регулярности. Алгебрами такого класса являются, в частности, линейные структуры (структуры Ли-Березина-Кириллова) на дуальных пространствах полупростых алгебр Ли, квадратичные эллиптические алгебры Склянина, а также полиномиальные алгебры, недавно описанные Бондалом, Дубровиным и Угальей. В этих алгебрах найдены простые детерминантные соотношения между скобками и операторами Казимира. Эти соотношения, в частности, устанавливают, что сумма степеней операторов Казимира совпадает с размерностью алгебры для эллиптических алгебр Склянина. Приводятся примеры таких алгебр и показано, что некоторые из них естественным образом возникают в гамильтоновых интегрируемых системах. Среди этих примеров находится и новый класс двухчастичных интегрируемых систем, зависяший эллиптическим образом как от координат, так и от импульсов.

Ключевые слова: полиномиальные пуассоновы структуры, эллиптические алгебры, интегрируемые системы.

\section{1. ВВЕДЕНИЕ}

В данной статье полиномиальными пуассоновыми структурами называются те структуры, скобки Пуассона для которых суть многочлены от локальных координат на подлежашем пуассоновом многообразии. Типичный пример такой структуры - это знаменитая алгебра Склянина [1], [2].

Напомним, что пуассонова структура на многообразии $M$ (гладком или алгебраическом) задается бивекторным антисимметричным тензорным полем $\pi \in \Lambda^{2}(T M)$, которое порождает структуру (возможно, бесконечномерной) алгебры Ли на соответствующем пространстве функций на $M$, задаваемую скобками Пуассона

$$
\{f, g\}=\langle\pi, d f \wedge d g\rangle .
$$

\footnotetext{
* Институт теоретической физики им. Л. Д. Ландау, Москва, Россия; Dépt. de Mathématiques, Univ. d'Angers, Angers, France

${ }^{\dagger}$ Dépt. de Mathématiques, Univ. d'Angers, Angers, France; Институт теоретической и экспериментальной физики, Москва, Россия
} 
Тождество Якоби для этих скобок эквивалентно некоторому аналогу (классического) уравнения Янга-Бакстера, а именно пуассонову мастер-уравнению $[\pi, \pi]=0$, в котором скобки $[\cdot, \cdot]: \Lambda^{p}(T M) \times \Lambda^{q}(T M) \mapsto \Lambda^{p+q-1}(T M)$ являются единственно возможной структурой супералгебры Ли на $\Lambda^{*}(T M)$, задаваемой так называемыми скобками Схоутена. Для более подробного ознакомления со всеми приведенными сведениями можно порекомендовать, например, книгу [3].

В работе [4] был найден результат, аналогичный теореме Дарбу, который позволяет дать локальное описание любого пуассонова многообразия. А именно, всегда возможно выбрать координаты $\left(q_{1}, \ldots, q_{l}, p_{1}, \ldots, p_{l}, x_{1}, \ldots, x_{k}\right)$ в окрестности произвольной точки $m \in M$, в которых бивекторное поле $\pi$ принимает вид

$$
\pi=\sum_{i=1}^{l} \frac{\partial}{\partial q_{i}} \wedge \frac{\partial}{\partial p_{i}}+\sum_{i>j}^{k} f_{i j}(x) \frac{\partial}{\partial x_{i}} \wedge \frac{\partial}{\partial x_{j}}
$$

где $f_{i j}(m) \equiv 0$.

Случай $k=0$ отвечает симплектической структуре, в то время как противоположный случай $l=0$ обычно соответствует полностью вырожденной пуассоновой структуpe. Напомним важное понятие функций Казимира структуры $\pi$. Функция $F \in \operatorname{Fun}(M)$ представляет собой оператор Казимира пуассоновой структуры $\pi$, если $\{F, G\}=0$ при всех функциях $G \in \operatorname{Fun}(M)$. Ясно, что если ранг структуры постоянен в окрестности точки $m$ (тогда $m$ называется регулярной точкой), то операторы Казимира в этой окрестности суть функции, зависящие только от $x_{1}, \ldots, x_{k}$, а пуассоново многообразие допускает расслоение на симплектические листы, т.е. такое многообразие представимо в виде объединения подмногообразий $x_{1}=c_{1}, \ldots, x_{k}=c_{k}$, где $c_{i}$ постоянны, а структура $\pi$ невырожденна на каждом симплектическом листе. Вообще говоря, размерность листов постоянна только на плотном открытом подмножестве регулярных точек и может варьироваться вне этого подмножества.

В данной статье рассматриваются полиномиальные пуассоновы структуры, симплектические листы которых обладают некоторыми свойствами регулярности. Сейчас воздержимся от строгого определения этих свойств и отметим лишь то, что этим свойствам удовлетворяет цельй ряд систем с интересными классами пуассоновых структур, наиболее известной из которых является знаменитая пуассонова структура Ли-БерезинаКириллова на пространстве $\mathfrak{g}^{*}$, дуальном к полупростой алгебре Ли $\mathfrak{g}$. Эта структура линейна относительно координатных функций $x_{1}, \ldots, x_{n}$ на $\mathfrak{g}^{*}$, отвечающих базису $X_{1}, \ldots, X_{n}$ на $\mathfrak{g}$, и она задается структурными постоянными на $\mathfrak{g}$. Наоборот, всякая линейная структура $\left\{x_{i}, x_{j}\right\}=C_{i j}^{k} x_{k}$ возникает из некоторой алгебры Ли. Регулярные пуассоновы скобки Ли-Березина-Кириллова получаются, если рассмотреть полупростые алгебры Ли.

Другой широкий класс содержательных пуассоновых структур с регулярными симплектическими листами содержится в подклассе квадратичны $x$ пуассоновых алгебр, введенных Скляниным [5] и описанных в работах [1], [2] в более общем контексте, как квазиклассический предел ассоциативных алгебр с квадратичными соотношениями, которые представляют собой плоские деформации алгебры функций на $\mathbb{C}^{n}$. Эти алгебры ассоциированы с эллиптическими кривыми и обладают следующим интригующим 
свойством: для таких алгебр операторы Казимира полиномиальны, и во всех таких алгебрах размерность соответствуюшей алгебры равна сумме показателей степеней генераторов кольца многочленов операторов Казимира. Одна из задач данной работы состоит в том, чтобы объяснить это свойство, которое будет следовать из простых детерминантных соотношений между альтернированными произведениями скобок Пуассона для функций от координат и минорами матрицы Якоби для операторов Казимира, выраженных через эти координаты (см. теорему 3.1).

Другой мотив, побуждаюший к исследованию такого типа полиномиальных пуассоновых алгебр, связан с гамильтоновыми интегрируемыми системами. Будет показано, что такие пуассоновы алгебры возникают естественным образом как гамильтоновы структуры, ассоциированные с широким спектром физически значимых моделей: от простого волчка Арнольда-Эйлера-Нама до недавно открытой дваждыэллиптической $S U(2)$-модели [6], [7]. Полиномиальная пуассонова алгебра, которая порождает естественную гамильтонову структуру этой модели, однородна и имеет максимальную степень 3.

Статья организована следуюшим образом. В разделе 2 приводятся общие сведения, касающиеся полиномиальных пуассоновых алгебр. Даются некоторые известные (и не так хорошо известные) определения, обсуждается голоморфное продолжение аффинных пуассоновых структур и дается описание обобщенных эллиптических алгебр Склянина [1].

В разделе 3 определяются алгебры с регулярной структурой симплектических листов и доказывается теорема 3.1 (доказательство представляет собой прямое вычисление). Менее очевидный пример, в котором утверждение теоремы 3.1 остается справедливым, дается обобщенной алгеброй Склянина $q_{5}$.

В разделах 4 и 5 содержатся различные примеры обсуждаемой конструкции. В разделе 4 приводятся весьма примечательные неполиномиальные замены переменных, которые тем не менее сохраняют полиномиальный вид пуассоновых структур, ассоциированных с эллиптическими кривыми. Эти замены переменных связаны с так называемой зеркальной симметрией многообразий Калаби-Яу. Явный вид и возможное значение таких замен в квантовом подходе работы [1] остаются интересной открытой проблемой. В разделе 5 приводятся примеры полиномиальных пуассоновых структур, ассоциированных с поверхностями $K 3$ (как гладкими, так и сингулярными). Интересным свойством этих структур является то, что все они задаются многочленами третьей степени от координат, и остается до сих пор непонятно, как можно "проквантовать" эти структуры или же как выглядят аналоги обобшенных алгебр Склянина в данном случае. Такие аналоги (если только они вообще сушествуют) могли бы называться "алгебрами Мукаи". В настояшее время известны только два примера таких алгебр: квантовые проективные прямые [8] и некоторые "некоммутативные" поверхности $K 3$ (последние получаются как результат деформации тороидальных орбифолдов [9], а потому не порождают никакого интересного примера квантования пуассоновой алгебры).

Наконец, раздел 6 содержит примеры некоторых естественных интегрируемых систем, являюшихся гамильтоновыми по отношению к обсуждаемым регулярным пуассоновым структурам. На самом деле одна из таких систем, а именно дваждыэллиптическая система, или система "DELL", которая имеет третий порядок в естественном 
гамильтоновом описании, и послужила мотивом, побуждающим к тому, чтобы дать по возможности общее описание таких структур. Подробное исследование этого интересного примера, связанного с шестимерной суперсимметричной калибровочной теорией с гипермультиплетами материи, будет дано в нашей совместной статье с Горским и Браденом. В настояшей же работе исследуются полиномиальные пуассоновы структуры, связанные с дваждыэллиптическими системами. Это частично объясняет в достаточной степени иллюстративный характер данной статьи. Достаточно часто будут приводиться вполне очевидные примеры с целью сделать содержание статьи доступным широкому кругу читателей (прежде всего, принадлежащих физическому сообществу).

\section{2. ПУАССОНОВЫ АЛГЕБРЫ НАД КОЛЬЦОМ МНОГОЧЛЕНОВ}

2.1. Общие замечания. В этом разделе описываются полиномиальные пуассоновы алгебры, заданные на аффинной части некоторых алгебраических переменных. Как правило, эти переменные вкладываются как (полные) пересечения во (взвешенные) проективные пространства. Построение переменных пересечения во взвешенных проективных пространствах довольно часто оказывается эквивалентным процедуре построения пересечений в произведениях стандартных пространств $\mathbb{C} P^{n}-$ факт, хорошо известный специалистам струнной теории, которые интерпретируют полные пересечения, приводящие к многообразиям Калаби-Яу, как сценарий компактификации для суперструнного вакуума, а зеркальная симметрия при этом служит отображением между пространствами модулей взвешенных многообразий Калаби-Яу (см. [10], [11]).

Рассмотрим $n-2$ многочленов $Q_{i}$ над пространством $\mathbb{C}^{n}$ с координатами $x_{i}, \quad i=$ $1, \ldots, n$. Для любого многочлена $\lambda \in \mathbb{C}\left[x_{1}, \ldots, x_{n}\right]$ зададим билинейную дифференциальную операцию

$$
\{\cdot, \cdot\}: \mathbb{C}\left[x_{1}, \ldots, x_{n}\right] \otimes \mathbb{C}\left[x_{1}, \ldots, x_{n}\right] \mapsto \mathbb{C}\left[x_{1}, \ldots, x_{n}\right]
$$

с помощью формулы

$$
\{f, g\}=\lambda \frac{d f \wedge d g \wedge d Q_{1} \wedge \cdots \wedge d Q_{n-2}}{d x_{l} \wedge d x_{2} \wedge \cdots \wedge d x_{n}}, \quad f, g \in \mathbb{C}\left[x_{1}, \ldots, x_{n}\right] .
$$

Эта операция задает структуру пуассоновой алгебры над $\mathbb{C}\left[x_{1}, \ldots, x_{n}\right]$ как частный случай более общей $(n-m)$-арной операции Намбу, задаваемой антисимметричным $(n-m)$-поливекторным полем $\eta$,

$$
\left\langle\eta, d f_{1} \wedge \cdots \wedge d f_{n-m}\right\rangle=\left\{f_{1}, \ldots, f_{n-m}\right\}
$$

зависящим от $m$ полиномиальных "операторов Казимира" $Q_{1}, \ldots, Q_{m}$ и от $\lambda$ :

$$
\left\{f_{1}, \ldots, f_{n-m}\right\}=\lambda \frac{d f_{1} \wedge \cdots \wedge d f_{n-m} \wedge d Q_{1} \wedge \cdots \wedge d Q_{m}}{d x_{l} \wedge d x_{2} \wedge \cdots \wedge d x_{n}}, \quad f_{i} \in \mathbb{C}\left[x_{1}, \ldots, x_{n}\right] .
$$

Здесь операция

$$
\{\cdot, \ldots, \cdot\}: \mathbb{C}\left[x_{1}, \ldots, x_{n}\right]^{\otimes n-m_{\mapsto}} \mathbb{C}\left[x_{1}, \ldots, x_{n}\right]
$$

такова, что должны быть выполнены следующие три свойства: 
1) антисимметричность

$$
\left\{f_{1}, \ldots, f_{n-m}\right\}=(-1)^{\sigma}\left\{f_{\sigma(1)}, \ldots, f_{\sigma(n-m)}\right\}, \quad \sigma \in \operatorname{Symm}_{n-m} ;
$$

2) покоординатное правило Лейбница, которое утверждает, что для любой функции $h \in \mathbb{C}\left[x_{1}, \ldots, x_{n}\right]$ имеет место соотношение

$$
\left\{f_{1} h, \ldots, f_{n-m}\right\}=f_{1}\left\{h, \ldots, f_{n-m}\right\}+h\left\{f_{1}, \ldots, f_{n-m}\right\} ;
$$

3) фундаментальное тождество (которое заменяет собой тождество Якоби):

$$
\begin{aligned}
\left\{\left\{f_{1}, \ldots, f_{n-m}\right\}, f_{n-m+1}, \ldots, f_{2(n-m)-1}\right\}+ \\
\quad+\left\{f_{n-m},\left\{f_{1}, \ldots, f_{n-m-1}, f_{n-m+1}\right\}, f_{n-m+2}, \ldots, f_{2(n-m)-1}\right\}+\cdots \\
\\
\quad \cdots+\left\{f_{n-m}, \ldots, f_{2(n-m)-2},\left\{f_{1}, \ldots, f_{n-m-1}, f_{2(n-m)-1}\right\}\right\}= \\
=\left\{f_{1}, \ldots, f_{n-m-1},\left\{f_{n-m}, \ldots, f_{2(n-m)-1}\right\}\right\},
\end{aligned}
$$

оно должно выполняться для любых функций $f_{1}, \ldots, f_{2(n-m)-1} \in \mathbb{C}\left[x_{1}, \ldots, x_{n}\right]$.

Такое естественное обобщение пуассоновой структуры (которая сама отвечает случаю $n-m=2$ ) было введено Намбу [12] и впоследствии всесторонне исследовано Тахтаджяном [13].

Наиболее естественным примером структуры Намбу-Пуассона является так называемая каноническая структура Намбу-Пуассона на $\mathbb{C}^{m}$ с координатами $x_{1}, \ldots, x_{m}$ :

$$
\left\{f_{1}, \ldots, f_{m}\right\}=\operatorname{Jac}\left(f_{1}, \ldots, f_{m}\right)=\frac{\partial\left(f_{1}, \ldots, f_{m}\right)}{\partial\left(x_{1}, \ldots, x_{m}\right)} .
$$

Отметим, что соотношение (1) для скобок Пуассона также выполняется и в более обшем случае, когда многочлены $Q_{i}$ заменяются на рациональные функции, но получившиеся скобки по-прежнему остаются полиномиальными. В действительности эта формула справедлива в кольце степенных рядов.

Многочлены $Q_{i}, i=1, \ldots, n-2$, суть операторы Казимира для скобки (1), и всякая пуассонова структура в $\mathbb{C}^{n} \mathrm{c} n-2$ операторами Казимира $Q_{i}$ в случае обшего положения может быть представлена в указанном виде.

Вышеприведенная конструкция отнюдь не нова и широко известна еше с работ Намбу и Склянина. Интерес к этой конструкции снова возник совсем недавно [14], [15] в другом контексте, связанном с общим интересом к проблеме классификации и квантования пуассоновых структур.

Случай $n=4$ в формуле (1) отвечает классической (обобщенной) квадратичной пуассоновой алгебре Склянина. Собственно алгебра Склянина получается из следуюших двух квадрик в $\mathbb{C}^{4}$ :

$$
\begin{aligned}
& Q_{1}=x_{1}^{2}+x_{2}^{2}+x_{3}^{2}, \\
& Q_{2}=x_{4}^{2}+J_{1} x_{1}^{2}+J_{2} x_{2}^{2}+J_{3} x_{3}^{2} .
\end{aligned}
$$

Скобки Пуассона (1) афффинных координат с $\lambda=1$ имеют вид

$$
\left\{x_{i}, x_{j}\right\}=(-1)^{i+j} \operatorname{det}\left(\frac{\partial Q_{k}}{\partial x_{l}}\right), \quad l \neq i, j, \quad i>j .
$$


Заметим, что выражение (1) обладает преимушествами перед выражением (5), поскольку первое выражение оказывается совместным с более общей ситуацией, когда пересекаюшиеся переменные погружаются во взвешенные проективные пространства или в произведение проективных пространств. Ниже рассматривается пример такой конструкции.

Естественным образом встает проблема расширить скобки (1) или (5) с пространства $\mathbb{C}^{n}$ на проективное пространство $\mathbb{C} P^{n}$.

ПРЕДЛОЖЕНИЕ 2.1. Пусть $X_{1}, \ldots, X_{n}$ суть координаты на пространстве $\mathbb{C}^{n}$, интерпретированном как аффинная часть соответствующего проективного пространства $\mathbb{C} P^{n}$ с заданными однородными координатами $\left(x_{0}: x_{1}: \ldots: x_{n}\right), X_{i}=$ $x_{i} / x_{0}$. Если скобка $\left\{X_{i}, X_{j}\right\}$ может бъть расширена до голоморфной пуассоновой структуры на $\mathbb{C} P^{n}$, то максимальная степень этой структуры (т.е. максимальная степень мономов в $\left.X_{i}\right)$ равна трем, и должно выполняться тождество

$$
X_{k}\left\{X_{i}, X_{j}\right\}_{3}+X_{i}\left\{X_{j}, X_{k}\right\}_{3}+X_{j}\left\{X_{k}, X_{i}\right\}_{3}=0, \quad i \neq j \neq k
$$

m.e. $\left\{X_{i}, X_{j}\right\}_{3}=X_{i} Y_{j}-X_{j} Y_{i}$, əде $\operatorname{deg} Y_{i}=2$.

ДокАЗАТЕЛЬСТВо. После замены координат получим

$$
\left\{\frac{1}{X_{i}}, \frac{X_{j}}{X_{i}}\right\}=-\frac{1}{X_{i}^{3}}\left\{X_{i}, X_{j}\right\},
$$

а потому $\operatorname{deg}\left\{X_{i}, X_{j}\right\} \leqslant 3$. Более того, можно заключить, что

$$
\left\{X_{i}, X_{j}\right\}=\left\{X_{i}, X_{j}\right\}_{0}+\left\{X_{i}, X_{j}\right\}_{1}+\left\{X_{i}, X_{j}\right\}_{2}+\left\{X_{i}, X_{j}\right\}_{3},
$$

где $\operatorname{deg}\left\{X_{i}, X_{j}\right\}_{k}=k, k=0,1,2,3$. Формула (6) следует из тождеств

$$
\left\{\frac{X_{j}}{X_{i}}, \frac{X_{k}}{X_{i}}\right\}=\frac{1}{X_{i}^{2}}\left\{X_{j}, X_{k}\right\}-\frac{X_{k}}{X_{i}^{3}}\left\{X_{j}, X_{i}\right\}-\frac{X_{j}}{X_{i}{ }^{3}}\left\{X_{i}, X_{k}\right\},
$$

и утверждение предложения тем самым доказано.

В обшем случае однородные пуассоновы алгебры, описываемые структурами (1) или (5), не допускают проективных расширений, поскольку они не удовлетворяют условиям предложения 2.1 при $n \geqslant 4$. Пуассоновы алгебры вида (1) или (5) обладают тем свойством, что их симплектические листы имеют "низшие" размерности (размерность ноль или два). Приведенное ниже описание структур симплектических листов "низших" размерностей следует непосредственно из определения симплектических листов.

ПРЕДЛОЖЕНИЕ 2.2. Пусть $\left\{x_{i}, x_{j}\right\}=\left\langle\pi, d x_{i} \wedge d x_{j}\right\rangle=p_{i j}$ - аффинная пуассонова структура. Тогда $\pi$ мохсет иметь лишь симплектические листь “низиих" размерностей тогда и только тогда, когда $p_{i j}$ мохет быть представлена в плюккеровской форме, т.е. $p_{i j}=\alpha_{i} \beta_{j}-\alpha_{j} \beta_{i}$, где $\alpha_{i}, \beta_{j}-$ некоторые функции (не обязательно многочлены). 
2.2. Пуассоновы алгебры, ассоциированные с эллиптическими кривыми. Другой широкий класс полиномиальных пуассоновых алгебр возникает как квазиклассический предел $q_{n, k}(\mathcal{E})$ ассоциативных квадратичных алгебр $Q_{n, k}(\mathcal{E}, \eta)$, которые были описаны в работах [1], [2]. Здесь $\mathcal{E}$ обозначает эллиптическую кривую, а $n$ и $k-$ целые числа, не имеющие общих делителей и такие, что $1 \leqslant k<n$, в то время как $\eta-$ комплексное число, а $Q_{n, k}(\mathcal{E}, 0)=\mathbb{C}\left[x_{1}, \ldots, x_{n}\right]$.

Обозначим через $\mathcal{E}=\mathbb{C} / \Gamma$ эллиптическую кривую, заданную решеткой $\Gamma=\mathbb{Z} \oplus \tau \mathbb{Z}$, где $\tau \in \mathbb{C}, \operatorname{Im} \tau>0$. Генераторами алгебры $Q_{n, k}(\mathcal{E}, \eta)$ являются элементы $x_{i}, \quad i \in$ $\mathbb{Z} / n \mathbb{Z}$, на которые наложены условия

$$
\sum_{r \in \mathbb{Z} / n \mathbb{Z}} \frac{\theta_{j-i+r(k-1)}(0)}{\theta_{j-i-r}(-\eta) \theta_{k r}(\eta)} x_{j-r} x_{i+r}=0
$$

и которые удовлетворяют следуюшим свойствам:

1) $Q_{n, k}(\mathcal{E}, \eta)=\mathbb{C} \oplus Q_{1} \oplus Q_{2} \oplus \cdots$ так, что $Q_{\alpha} * Q_{\beta}=Q_{\alpha+\beta}$ (где символом $*$ обозначено умножение в алгебре), т.е. алгебры $Q_{n, k}(\mathcal{E}, \eta)$ имеют $\mathbb{Z}$-градуировку;

2 ) функция Гильберта многочленов $Q_{n, k}(\mathcal{E}, \eta)$ имеет вид

$$
\sum_{\alpha \geqslant 0} \operatorname{dim} Q_{\alpha} t^{\alpha}=\frac{1}{(1-t)^{n}} .
$$

Таким образом, можно видеть, что алгебра функций $Q_{n, k}(\mathcal{E}, \eta)$ при фиксированной кривой $\mathcal{E}$ представляет собой плоскую деформацию кольца многочленов $\mathbb{C}\left[x_{1}, \ldots, x_{n}\right]$.

Пусть $q_{n, k}(\mathcal{E})$ представляет собой соответствующую пуассонову алгебру. В работе [1] было показано, что алгебра $q_{n, k}(\mathcal{E})$ имеет $l=\operatorname{gcd}(n, k+1)$ операторов Казимира. Обозначим их через $P_{\alpha}, \alpha \in \mathbb{Z} / l \mathbb{Z}$. При этом их степени $\operatorname{deg} P_{\alpha}=n / l$.

Подчеркнем, что алгебры $q_{n, k}(\mathcal{E})$ при $n>4$ допускают симплектические листы размерностей, бо́льших двух, а потому эти алгебры не могут быть заданы с помощью формул $(1),(5)$. Примеры алгебр $q_{3,1}(\mathcal{E}), q_{4,1}(\mathcal{E})$ и $q_{5,1}(\mathcal{E})$ даются ниже.

\section{3. АЛГЕБРЫ С РЕГУЛЯРНЫМИ СТРУКТУРАМИ СИМПЛЕКТИЧЕСКИХ ЛИСТОВ}

Напомним, что минимальная коразмерность симплектических листов пуассоновой алгебры называется рангом этой алгебры. В настоящем разделе описывается класс пуассоновых алгебр на $\mathbb{C}^{n}$, удовлетворяющих следующим свойствам регулярности.

ОПреДЕлЕниЕ 3.1. Полиномиальная пуассонова алгебра $A$ ранга $l$ на $\mathbb{C}^{n}$ называется алгеброй с регулярной структурой симплектических листов, если

$1)$ центр $Z(A)$ данной алгебры есть кольцо многочленов $\mathbb{C}\left[Q_{1}, \ldots, Q_{l}\right]$ от операторов Казимира $Q_{i}$;

2) подмногообразие

$$
L_{\lambda_{1}, \ldots, \lambda_{l}}:=\bigcap_{i}\left\{Q_{i}=\lambda_{i}\right\}
$$

является полным пересечением (или, другими словами, касательные пространства $T_{p}\left(\left\{Q_{i}=\lambda_{i}\right\}\right)$ пересекаются трансверсально в точке общего положения $\left.p \in L_{\lambda_{1}, \ldots, \lambda_{l}}\right)$; 
3) если $M^{*}$ представляет собой объединение $\bigcup_{i}\left\{F_{i}\right\}$ симплектических листов размерностей $\operatorname{dim} F_{i}<n-l$, то $\operatorname{dim} M^{*} \leqslant n-2$;

4) если $L^{*}$ может быть представлено как объединение сингулярных подмногообразий $L_{\lambda_{1}, \ldots, \lambda_{l}}$ при различных $\lambda_{1}, \ldots, \lambda_{l}$, то $\operatorname{dim} L^{*} \leqslant n-2$.

Эллиптические алгебры $q_{n, k}(\mathcal{E})$ удовлетворяют этим условиям, как следует из описания их симплектических листов, данного в работе [2]. Все эллиптические алгебры, равно как и все прочие алгебры, которые удовлетворяют условиям определения 3.1, удовлетворяют также и следуюшей теореме.

Теорема 3.1. Имеем

$$
\underbrace{\pi \wedge \pi \wedge \cdots \wedge \pi}_{(n-l) / 2}=\lambda\left(d Q_{1} \wedge \cdots \wedge d Q_{l}\right)^{*}, \quad \lambda \in \mathbb{C}^{*},
$$

где $l$ - размерность пуассонова иентра (число операторов Казимира), $Q_{1}, \ldots, Q_{l}$ - операторы Казимира, а индекс * обозначает дуальность межсду l-формами и l-поливекторами, определяемую стандартной формой обвема $d x_{1} \wedge \cdots \wedge d x_{n}$.

Выражение в правой части уравнения (7) может быть переписано в координатном виде как

$$
\lambda \operatorname{det}\left(\begin{array}{ccc}
\frac{\partial Q_{1}}{\partial x_{i_{n-l+1}}} & \cdots & \frac{\partial Q_{1}}{\partial x_{i_{n}}} \\
\vdots & \ddots & \vdots \\
\frac{\partial Q_{l}}{\partial x_{i_{n-l+1}}} & \cdots & \frac{\partial Q_{l}}{\partial x_{i_{n}}}
\end{array}\right)=\operatorname{Alt}_{\left(i_{1}, \ldots, i_{n}\right)}\left(\left\{x_{i_{1}}, x_{i_{2}}\right\} \ldots\left\{x_{i_{n-l-1}}, x_{i_{n-l}}\right\}\right),
$$

где $\left(i_{1}, \ldots, i_{n}\right)$ - четная перестановка $(1, \ldots, n)$, а $\lambda \in \mathbb{C}^{*}$ - ненулевая постоянная.

ДокАЗАТЕЛЬСТво. Для произвольных $n-l$ полиномиальных функций $f_{1}, \ldots, f_{n-l}$ формулу (7) можно переписать в виде

$$
w^{(n-l) / 2}\left(f_{1}, \ldots, f_{n-l}\right)=\lambda \frac{d f_{1} \wedge \cdots \wedge d f_{n-l} \wedge d Q_{1} \wedge \cdots \wedge d Q_{l}}{d x_{1} \wedge \cdots \wedge d x_{n}}, \quad \lambda \in \mathbb{C}^{*} .
$$

Обозначим через $\Omega_{1}$ и $\Omega_{2}$ соответственно левую и правую части уравнения (8). Ясно, что $\imath_{Q_{\alpha}} \Omega_{i}=0$ для каждого $\alpha, i=1,2$, а потому, выражая тензоры $\Omega_{i}$ через координаты,

$$
\Omega_{i}=\sum_{\left(\alpha_{1}, \ldots, \alpha_{n-l}\right)} w_{\alpha_{1}, \ldots, \alpha_{l}}^{i} \partial_{\alpha_{1}} \wedge \cdots \wedge \partial_{\alpha_{n-l}},
$$

можно получить систему линейных уравнений на коэффициенты $w_{\alpha_{1}, \ldots, \alpha_{l}}^{i}$ :

$$
\imath_{Q_{\alpha}} \Omega_{i}=\sum_{\alpha_{1}} w_{\alpha_{1}, \ldots, \alpha_{n-l}}^{i} Q_{\alpha x_{\alpha_{1}}}^{\prime} \partial_{\alpha_{2}} \wedge \cdots \wedge \partial_{\alpha_{n-l}}=0
$$

Из этой системы можно получить, что обе части уравнения (8) пропорциональны друг другу ( $\lambda$ - функция, не обрашающаяся тождественно в нуль). Функция $\lambda$ рациональна, а потому существуют два многочлена $p_{1}$ и $p_{2}$ без общих делителей такие, что $\lambda=$ $p_{1} / p_{2}$. Но первый многочлен $p_{1}$ имеет набор нулей, лежащих в области $M^{*}$, и потому $\operatorname{codim} M^{*}=1$. Принимая во внимание условие 3 определения 3.1 , получим, что $p_{1}=$ const.

Аналогичные рассуждения показывают, что нули многочлена $p_{2}$ принадлежат (или совпадают) множеству $L^{*}$, а потому $p_{2}=$ const. Теорема доказана. 
ЗАМЕчАНИЕ 3.1. Утверждение теоремы 3.1 остается справедливым для любой пуассоновой алгебры ранга $l \mathrm{c} l$ независимыми центральными элементами $Q_{\alpha}$, если позволить общему множителю $\lambda$ принимать вид произвольной рациональной функции.

ЗАмечАниЕ 3.2. Если $A$ есть квадратичная пуассонова алгебра, то в соответствии с условиями теоремы 3.1 справедливо следуюшее соотношение между суммарной степенью операторов Казимира и размерностью $A$ :

$$
\sum_{\alpha} \operatorname{deg} Q_{\alpha}=\operatorname{dim} A=n .
$$

Более того, для всех известных эллиптических алгебр, которые представляют собой градуированные деформации $\mathbb{Z}^{n}$-градуированного кольца многочленов с конечным набором генераторов, сумма степеней всех этих генераторов равна сумме степеней всех операторов Казимира. Например,

$$
n=\sum_{i \in \mathbb{Z} / n \mathbb{Z}} \operatorname{deg} x_{i}=\sum_{j \in \mathbb{Z} / l \mathbb{Z}} \operatorname{deg} Q_{j} .
$$

ЗАмЕчАниЕ 3.3 . В случае, когда условие 3 определения 3.1 не имеет места, может быть полезным следующее определение.

ОПреДЕЛЕнИЕ 3.2. Элемент $Q \in A$ называется квазицентральныц или квазиоператором Казимира, если скобка $\{Q, f\}$ делится на $Q$ для любого $f \in A$.

Ясно, что если $Q_{1}$ и $Q_{2}$ - квазицентральные элементы алгебры $A$, то их произведение $Q_{1} Q_{2}$ также представляет собой квазицентральный элемент. Пусть $\operatorname{codim} M^{*}=1$ и $M_{i}$ суть неприводимые компоненты $M^{*}$ размерности $n-1$. Для компонент $M_{i}$, задаваемых уравнениями $Q_{i}=0$, элементы $Q_{i}$, очевидно, являются квазиоператорами Казимира. Обратно, если неразложимый оператор $Q$ представляет собой квазицентральный, но не центральный элемент, то гиперповерхность $Q=0$ содержится в $M^{*}$.

Последнее интересное и, возможно, полезное наблюдение состоит в том, что скобка $\{\ln Q, \cdot\}$ является (внешней) производной $A$ для каждого квазицентрального элемента $Q$.

Для того чтобы придать некоторый вес последней теореме, покажем, что она справедлива для алгебры $q_{5}(\mathcal{E})$ из работы [1].

ПримеР. Рассмотрим кольцо многочленов с пятью генераторами $x_{i}, i \in \mathbb{Z} / 5 \mathbb{Z}$, на которые наложены следуюшие скобки Пуассона:

$$
\begin{aligned}
& \left\{x_{i+1}, x_{i+4}\right\}=-\frac{1}{5}\left(k^{2}+\frac{3}{k^{3}}\right) x_{i+1} x_{i+4}+2 x_{i+2} x_{i+3}+k x_{i}^{2}, \\
& \left\{x_{i+2}, x_{i+3}\right\}=\frac{1}{5}\left(3 k^{2}-\frac{1}{k^{3}}\right) x_{i+2} x_{i+3}+\frac{2}{k} x_{i+1} x_{i+4}-\frac{1}{k^{2}} x_{i}^{2},
\end{aligned}
$$


где $k \in \mathbb{C}$ есть параметр кривой $\mathcal{E}_{\tau}=\mathbb{C} / \Gamma$, т.е. некоторая функция от $\tau$. Центр $Z\left(q_{5}(\mathcal{E})\right)$ порождается многочленом

$$
\begin{aligned}
P= & -\frac{1}{k}\left(x_{0}^{5}+x_{1}^{5}+x_{2}^{5}+x_{3}^{5}+x_{4}^{5}\right)+ \\
& +\left(\frac{1}{k^{5}}-3\right)\left(x_{0}^{3} x_{1} x_{4}+x_{1}^{3} x_{0} x_{2}+x_{2}^{3} x_{1} x_{3}+x_{3}^{3} x_{2} x_{4}+x_{2}^{3} x_{0} x_{3}\right)+ \\
& +\left(k^{3}+\frac{3}{k^{3}}\right)\left(x_{0}^{3} x_{2} x_{3}+x_{1}^{3} x_{3} x_{4}+x_{2}^{3} x_{0} x_{4}+x_{3}^{3} x_{1} x_{0}+x_{4}^{3} x_{1} x_{2}\right)- \\
& -\left(2 k+\frac{1}{k^{4}}\right)\left(x_{0} x_{1}^{2} x_{4}^{2}+x_{1} x_{2}^{2} x_{0}^{2}+x_{2} x_{0}^{2} x_{4}^{2}+x_{3} x_{1}^{2} x_{0}^{2}+x_{4} x_{1}^{2} x_{2}^{2}\right)+ \\
& +\left(k^{2}-\frac{2}{k^{3}}\right)\left(x_{0} x_{2}^{2} x_{3}^{2}+x_{1} x_{3}^{2} x_{4}^{2}+x_{2} x_{0}^{2} x_{4}^{2}+x_{3} x_{1}^{2} x_{0}^{2}+x_{4} x_{1}^{2} x_{2}^{2}\right)+ \\
& +\left(k^{4}+\frac{16}{k}-\frac{1}{k^{6}}\right) x_{0} x_{1} x_{2} x_{3} x_{4} .
\end{aligned}
$$

Легко проверить, что для любого $i \in \mathbb{Z} / 5 \mathbb{Z}$

$\left\{x_{i+1}, x_{i+2}\right\}\left\{x_{i+3}, x_{i+4}\right\}+\left\{x_{i+3}, x_{i+1}\right\}\left\{x_{i+2}, x_{i+4}\right\}+\left\{x_{i+2}, x_{i+3}\right\}\left\{x_{i+1}, x_{i+4}\right\}=\frac{1}{5} \frac{\partial P}{\partial x_{i}}$.

\section{4. ПРИМЕРЫ АЛГЕБР С РЕГУЛЯРНЫМИ ЛИСТАМИ, $n=3$}

4.1. Эллиптические алгебры. Пусть

$$
P\left(x_{1}, x_{2}, x_{3}\right)=\frac{1}{3}\left(x_{1}^{3}+x_{2}^{3}+x_{3}^{3}\right)+k x_{1} x_{2} x_{3},
$$

тогда

$$
\begin{aligned}
& \left\{x_{1}, x_{2}\right\}=k x_{1} x_{2}+x_{3}^{2}, \\
& \left\{x_{2}, x_{3}\right\}=k x_{2} x_{3}+x_{1}^{2}, \\
& \left\{x_{3}, x_{1}\right\}=k x_{3} x_{1}+x_{2}^{2} .
\end{aligned}
$$

Квантовый аналог этой пуассоновой структуры есть алгебра $\mathcal{Q}_{3}(\mathcal{E}, \eta)$, где $\mathcal{E} \subset \mathbb{C} P^{2}-$ эллиптическая кривая $P\left(x_{1}, x_{2}, x_{3}\right)=0$.

4.2. Зеркальное преобразование. Интереснейшее свойство алгебры (10) состоит в том, что полиномиальный характер этой алгебры сохраняется при следуюших заменах переменных.

1. Пусть

$$
y_{1}=x_{1}, \quad y_{2}=x_{2} x_{3}^{-1 / 2}, \quad y_{3}=x_{3}^{3 / 2} .
$$

Многочлен $P^{\vee}$ в координатах $y_{1}, y_{2}, y_{3}$ принимает вид

$$
P^{\vee}\left(y_{1}, y_{2}, y_{3}\right)=\frac{1}{3}\left(y_{1}^{3}+y_{2}^{3} y_{3}+y_{3}^{2}\right)+k y_{1} y_{2} y_{3},
$$

а соответствуюшие скобки Пуассона опять полиномиальны (что совсем не очевидно!) и имеют тот же вид $\left\{y_{i}, y_{j}\right\}=(3 / 2)\left(\partial P^{\vee} / \partial y_{k}\right)$, где $i, j, k=1,2,3$. 
Если положить $\operatorname{deg} y_{1}=2, \operatorname{deg} y_{2}=1$ и $\operatorname{deg} y_{3}=3$, то многочлен $P^{\vee}$ оказывается также однороден по $y_{1}, y_{2}, y_{3}$ и задает при этом эллиптическую кривую $P^{\vee}=0$ во взвешенном проективном пространстве $\mathbb{W} P_{2,1,3}$.

2. Пусть теперь $z_{1}=x_{1}^{-3 / 4} x_{2}^{3 / 2}, z_{2}=x_{1}^{1 / 4} x_{2}^{-1 / 2} x_{3}$ и $z_{3}=x_{1}^{3 / 2}$. Многочлен $\widetilde{P}^{\vee}$ в координатах $z_{1}, z_{2}, z_{3}$ имеет вид

$$
\widetilde{P}^{\vee}\left(z_{1}, z_{2}, z_{3}\right)=\frac{1}{3}\left(z_{3}^{2}+z_{1}^{2} z_{3}+z_{1} z_{2}^{3}\right)+k z_{1} z_{2} z_{3},
$$

и соответствующие скобки Пуассона снова полиномиальны и имеют тот же самый вид $\left\{z_{i}, z_{j}\right\}=(9 / 4)\left(\partial \widetilde{P}^{\vee} / \partial z_{k}\right)$, где $i, j, k=1,2,3$.

Если положить $\operatorname{deg} z_{1}=1, \operatorname{deg} z_{2}=1$ и $\operatorname{deg} z_{3}=2$, то многочлен $\widetilde{P}^{\vee}$ опять оказывается однородным по $z_{1}, z_{2}, z_{3}$ и задает при этом эллиптическую кривую $\widetilde{P}^{\vee}=0$ во взвешенном проективном пространстве $\mathbb{W} P_{1,1,2}$.

Происхождение странной неполиномиальной замены переменных (11) восходит к конструкции зеркально-дуальных многообразий Калаби-Яу [10], для которых тор (12) зеркально-дуален тору (9). Зеркальное отображение тривиально для одномерных многообразий Калаби-Яу. Достаточно необычное наблюдение показывает, что отображение (11), которое становится пуассоновым, если выбрать подходяшее замыкание кольца многочленов неполиномиальными функциями, генерирует новое соотношение на квантовом уровне, а именно квантовую эллиптическую алгебру $Q_{3}\left(\mathcal{E}^{\vee}\right)$, которая отвечает оператору Казимира (12) и имеет параметр комплексной структуры $(\tau+1) / 3$, в то время как квантовая алгебра, отвечаюшая оператору Казимира (9), имеет параметр комплексной структуры $\tau$. Таким образом, эти две алгебры различны. "Квантовый" аналог отображения (11) до сих пор не найден, и этот вопрос заслуживает дальнейшего исследования.

4.3. Многочлены Маркова. Рациональные вырождения. Возьмем теперь в качестве оператора Казимира неоднородный многочлен (многочлен Маркова третьей степени) $P\left(x_{1}, x_{2}, x_{3}\right)=x_{1}^{2}+x_{2}^{2}+x_{3}^{2}+3 x_{1} x_{2} x_{3}$. Соответствуюшая пуассонова алгебpa $\left\{x_{i}, x_{j}\right\}=\partial P / \partial x_{k}, i, j, k=1,2,3$, с одной стороны, задает гамильтонову структуру на пространстве матриц Стокса в теории изомонодромных деформаций (см. [16]-[18]), а с другой стороны, описывает квазиклассический предел квантованных структур пространств Тейхмюллера [19]. Отметим, что обобшения этой алгебры на случай $n>3$, которые связаны с инвариантами старших степеней, впервые появились в работах Нельсон и Редже [20], были полностью исследованы Бондалом [21], который доказал, что симплектические листы этих алгебр имеют регулярную структуру.

Например, в соответствии с конструкцией Бондала-Дубровина-Угальи [17], [21] афффинная пуассонова алгебра в $\mathbb{C}^{6}$ с двумя операторами Казимира степеней 4 и 2, которая имеет четырехмерные симплектические листы на пересечении этих двух казимировских подмногообразий, удовлетворяет условиям определения 3.1 .

Отождествим $\mathbb{C}^{6}$ с шестимерным пространством матриц Стокса, имеющих вид

$$
\left(\begin{array}{llll}
1 & p & q & r \\
0 & 1 & x & y \\
0 & 0 & 1 & z \\
0 & 0 & 0 & 1
\end{array}\right) .
$$


Пуассонова алгебра матричных элементов содержит в правой части члены как первого, так и второго порядков (см. [21]).

Если выбрать два оператора Казимира в виде

$$
\begin{aligned}
& P_{1}=p z+x r-q y, \\
& P_{2}=p^{2}+q^{2}+r^{2}+x^{2}+y^{2}+z^{2}-p q x-p r y-q r z-x y z+p r x z,
\end{aligned}
$$

то можно непосредственно проверить, например, следуюшее соотношение:

$$
\begin{gathered}
\{x, y\}\{p, z\}+\{y, z\}\{p, x\}+\{z, x\}\{p, y\}=\operatorname{det}\left(\begin{array}{cc}
\frac{\partial P_{1}}{\partial q} & \frac{\partial P_{1}}{\partial r} \\
\frac{\partial P_{2}}{\partial q} & \frac{\partial P_{2}}{\partial r}
\end{array}\right)= \\
=\operatorname{det}\left(\begin{array}{cc}
-y & x \\
2 q-p x-r z & 2 r-p y-q z+p x z
\end{array}\right) .
\end{gathered}
$$

4.4. Полиномиальные расширения алгебры Аски-Вильсона. Классическая (пуассонова) алгебра Аски-Вильсона [22] описывается следуюшим полиномиальным оператором Казимира:

$$
P(x, y, z)=z^{2}-F(x, y), \quad F(x, y)=a x^{2} y^{2}+g(x, y),
$$

где $g(x, y)=a_{1} x^{2} y+a_{2} x y^{2}+a_{3} x^{2}+a_{4} y^{2}+a_{5} x y+a_{6} x+a_{7} y$. Квантовый аналог этой алгебры допускает естественное представление в терминах многочленов Аски-Вильсона.

Теперь легко построить полиномиальное расширение алгебры Аски-Вильсона, сохранив структуру операторов Казимира (14), но позволив функции $F(x, y)$ иметь произвольную степень. Например, если допустить, что функция $F(x, y)$ содержит в качестве членов максимальной степени мономы $x^{4}$ и $y^{4}$, то в этом случае получается расширение алгебры Аски-Вильсона, которое эквивалентно стандартной алгебре Склянина (3), (4) (см. [22]).

Предположим, что местоположение точек, удовлетворяющих условию $P(x, y, z)=0$, для выражения (14) представляет собой кривую во взвешенном проективном пространстве $\mathbb{W} P_{1,1,2}$, в котором переменные имеют степени $\operatorname{deg} x=\operatorname{deg} y=1$ и $\operatorname{deg} z=2$. При этом соотношение из замечания 3.2 по-прежнему остается справедливым.

\section{5. ПОВЕРХНОСТИ $K 3$}

5.1. Конус канонической кривой. Обозначим через $\mathcal{C}$ негиперэллиптическую кривую рода $g>1$. Пусть при этом $K_{\mathcal{C}}$ обозначает каноническое расслоение над кривой $\mathcal{C}$. Рассмотрим каноническое вложение $\xi: \mathcal{C} \rightarrow \mathbb{C} P^{g-1}$ и соответствующий конус $\mathbb{K}_{\mathcal{C}} \subset \mathbb{C}^{g}$ вложения $\xi(\mathcal{C})$. Кроме того, обозначим через $\widetilde{\mathbb{K}}_{\mathcal{C}}$ накрытие конуса $\mathbb{K}_{\mathcal{C}}$, которое отвечает накрытию $\mathcal{H} \mapsto \mathcal{E}$, где $\mathcal{H}$ - верхняя полуплоскость с координатой $\tau$ и $\widetilde{\mathbb{K}}_{\mathcal{C}}=$ $\mathcal{H} \times \mathbb{C}^{*}$. Обозначим через $d \tau$ соответствуюшую координату на $\mathbb{C}^{*}$. Хорошо известно, что $\mathbb{K}_{\mathcal{C}}=\widetilde{\mathbb{K}}_{\mathcal{C}} / \Gamma$, где $\Gamma \subset \mathfrak{s l}_{2}(\mathbb{R})$ - дискретная подгрупша, действуюшая естественным образом на координаты $\tau, d \tau$. Выберем пуассонову структуру на $\widetilde{\mathbb{K}}_{\mathcal{C}}$, имеюшую вид $\{\tau, d \tau\}=(d \tau)^{2}$. Легко проверить, что эта структура инвариантна относительно действия группы $\mathfrak{s l}_{2}(\mathbb{R})$ и обладает свойством симплектичности. А потому и конус $\mathbb{K}_{\mathcal{C}}$ оказывается однородным симплектическим многообразием. В случае $g=3$, конус $\mathbb{K}_{\mathcal{C}} \subset \mathbb{C}^{3}$ задается уравнением $p\left(x_{1}, x_{2}, x_{3}\right)=0$, где $p\left(x_{1}, x_{2}, x_{3}\right)$ - однородный многочлен четвертой степени. Введем на пространстве $\mathbb{C}^{3}$ полиномиальную пуассонову структуру с помошью тех же соотношений, что и выше: $\left\{x_{i}, x_{j}\right\}=\partial p / \partial x_{k}, i, j, k=1,2,3$. 
ПРЕДЛОЖЕНИЕ 5.1. Конус $\mathbb{K}_{\mathcal{C}} \subset \mathbb{C}^{3}$ есть симплектический лист выщеприведенной полиномиальной структурь, и ограничение этой структуры на конус $\mathbb{K}_{\mathcal{C}}$ имеет вид

$$
\{\tau, d \tau\}=\lambda(d \tau)^{2}, \quad \lambda \in \mathbb{C}^{*} .
$$

Аналогичные описания можно дать и в случаях $g=4,5$.

5.2. Общее обсуждение. Рассмотрим теперь компактификацию обсуждавшейся выше конструкции, получаюшуюся при добавлении одной точки к конусу, что эквивалентно замыканию с помошью проективного пространства $\mathbb{P}\left(K_{\mathcal{C}} \oplus \mathcal{O}_{\mathcal{C}}\right)$. При этом получается компактное двумерное комплексное многообразие с тривиальным антиканоническим расслоением, что эквивалентно голоморфному вложению кривой $C$ в поверхность $K 3$. Опишем теперь ограничение соответствующих пуассоновых структур на поверхность $K 3$, что дает симплектический лист таких структур. При этом мы получим интересный пример неквадратичных аффинных скобок.

На всех многообразиях $K 3$ сушествуют симплектические голоморфные формы, построенные Мукаи [23], и во всех примерах проективных моделей на $K 3$ можно реализовать симплектические скобки Мукаи как ограничения полиномиальных пуассоновых структур, взятых на пространствах $\mathbb{C}^{n}, n=3,4,5$, на подмногообразия, определенные как полные пересечения операторов Казимира этих полиномиальных пуассоновых структур. Соответствующие полиномиальные скобки имеют третий порядок. Заметим, что в соответствии с предложением 2.1 эти скобки не могут быть расширены до голоморфных пуассоновых структур на всем $\mathbb{C} P^{n}, n=3,4,5$. Ниже будет явно показано, что в данном случае действительно имеет место нетривиальное препятствие такому расширению.

Хорошо известно, что поверхности $K 3$ представляют собой единственные двумерные компактные комплексные многообразия, которые имеют нулевые первые числа Бетти и допускают сушествование голоморфной симплектической структуры. Ниже будет описано простое соотношение меж ду полиномиальными скобками Пуассона и конструкцией Мукаи голоморфной симплектической структуры на алгебраической поверхности $K 3$.

Конструкция Мукаи [23] основана на том простом обстоятельстве, что любое алгебраическое многообразие $K 3$ допускает проективное вложение в пространство $\mathbb{C} P^{n}$, на котором задана система однородных координат $\left(X_{0}: X_{1}: \ldots: X_{n}\right)$ и стандартная $n$-форма

$$
\Omega=\sum_{i=0}^{n}(-1)^{i} X_{i} d X_{0} \wedge \cdots \wedge \widehat{d X_{i}} \wedge \cdots \wedge d X_{n} .
$$

Эта форма используется для задания голоморфной 2-формы Мукаи с помощью формы вычетов $\operatorname{Res}_{\mathcal{S}}(\Omega / P)$ вдоль поверхности $K 3 \mathcal{S}$, где проективное вложение поверхности $\mathcal{S}$ задается местоположением нулей многообразия $P$, которое или представляет собой квартику $(n=3)$, или является трансверсальным пересечением квадрики и кубики $(n=4)$, или является трансверсальным пересечением трех квадрик $(n=5)$.

Можно непосредственно проверить, что полиномиальная пуассонова структура на $\mathbb{C}^{n}$, предъявленная выше при $n=3,4,5$, при ограничении на поверхность $\mathcal{S}$ совпадает со структурой Мукаи, записанной в аффинных координатах. 
5.3. Пример 1. Квартика Ферма. Положим, что $P_{4}\left(X_{0}, X_{1}, X_{2}, X_{3}\right)=0$, $\operatorname{deg} P_{4}=4$ (т.е. квартика), представляет собой поверхность $K 3$, вложенную в $\mathbb{C} P^{3}$. Выбирая открытую область $U_{0}=\left\{X_{0} \neq 0\right\}$, получим, что форма $\alpha=\Omega / X_{0}^{4}$, которая в своей аффинной части записывается как $\alpha_{0}=d x_{1} \wedge d x_{2} \wedge d x_{3}, x_{i}=X_{i} / X_{0}$, есть голоморфная 3-форма на $\mathcal{S} \backslash U_{0}$, и эта форма имеет простые полюсы вдоль пересечений $\mathcal{S} \cap U_{0}$. Следовательно, $\Omega / P=\alpha_{0} / P\left(1, x_{1}, x_{2}, x_{3}\right)$ в аффинной части, и вычет $\omega=\operatorname{Res} \mathcal{S} \Omega / P_{4}$ дается, например, формой

$$
\omega=\frac{d x_{1} \wedge d x_{2}}{\partial P_{4} / \partial x_{3}}
$$

и, как можно увидеть в аффинной карте $U_{0}$, соответствуюшая скобка дается выражением $\left\{x_{1}, x_{2}\right\}=-\partial P_{4} / \partial x_{3}$.

ПРЕДЛОЖЕНИЕ 5.2. Полиномиальные структуры на $\mathbb{C}^{n}, n=3,4,5$, задаваемые многочленом $P_{4}$ при $n=3$, полным пересечением квадрики $P_{2}$ и кубики $P_{3}$ при $n=4$ и пересечением трех квадрик $P_{2}, Q_{2}$ u $R_{2}$ при $n=5$, не имеют голоморфных расширений на проективнье пространства $\mathbb{C} P^{n}, \quad n=3,4,5$.

Чтобы доказать утверждение предложения 5.2, например, для случая $n=3$, можно показать, что, следуя предложению 2.1,

$X_{3}\left\{X_{1}, X_{2}\right\}_{3}+X_{1}\left\{X_{2}, X_{3}\right\}_{3}+X_{2}\left\{X_{3}, X_{1}\right\}_{3}=-\left(X_{3} \frac{\partial P_{4}}{\partial x_{3}}+X_{1} \frac{\partial P_{4}}{\partial x_{1}}+X_{2} \frac{\partial P_{4}}{\partial x_{2}}\right)=C \neq 0$.

Строго говоря, это рассуждение показывает только то, что структура, рассматриваемая на пространстве $K 3$, не может быть расширена с использованием данны $x$ полиномиальных формул. Можно также достаточно легко показать, что соответствующие скобки не допускают никаких расширений с поверхности $K 3$ на все пространство $\mathbb{C} P^{3}$. В самом деле, если бы такое расширение существовало, то оно обязательно было бы полиномиальным и совпадающим с исходными скобками на поверхности $K 3$; поэтому их разность обязана обращаться в нуль на поверхности, а потому должна быть как минимум четвертого порядка относительно аффинных координат, так как многочлен $P_{4}$ является делителем этой структуры.

5.4. Пример 2. Сингулярная поверхность $K 3$ в произведении проективных пространств. Другой интересный пример пуассоновой структуры на поверхности $K 3$ связан с так называемым принципом расщепления, восходящим к конструкции переменных Калаби-Яу и их зеркально-дуальных партнеров, изложенным в работе [10]. Грубо говоря, принцип расшепления позволяет рассматривать поверхность $K 3$ (возможно, сингулярную), которая вложена как гиперповерхность во взвешенное проективное пространство, как часть двумерного подмногообразия, вложенного в произведение стандартных (или в более обшем случае также взвешенных) проективных пространств. В таком случае можно использовать координатные формулы, а более строгий и концептуально богатый подход, как мы считаем, должен быть связан с подходяшим обобшением теории вычетов на случай торических переменных (см. [24]).

Следуя работе [25], рассмотрим гиперповерхность

$$
P=y_{1}\left(y_{1}^{3}+y_{3}^{6}+y_{5}^{3}\right)-y_{2}\left(y_{2}^{3}+y_{4}^{6}-y_{5}^{3}\right)=0,
$$


которая вложена в пространство $\mathbb{W} P_{1,1,2,2,2}[18]$ и становится сингулярной поверхностью $K 3$ в $\mathbb{C} P^{3}$

$$
P=x_{1}\left(x_{1}^{3}+x_{3}^{3}+x_{4}^{3}\right)-x_{2}\left(x_{2}^{3}+x_{3}^{3}-x_{4}^{3}\right)=0
$$

после переопределений $y_{4}=y_{3}$ и $x_{1}=y_{1}, x_{2}=y_{2}, x_{3}=y_{3}^{2}$ и $x_{4}=y_{5}$. В области $x_{1} \neq 0$ эта поверхность задается аффинным уравнением

$$
P=1+X_{3}^{3}+X_{4}^{3}-X_{2}^{4}-X_{2} X_{3}^{3}+X_{2} X_{4}^{3}=0,
$$

и в соответствии с конструкцией

$$
\left\{X_{2}, X_{3}\right\}=-\frac{\partial P}{\partial X_{4}}=-3 X_{4}^{2}\left(X_{2}+1\right) .
$$

Теперь эту поверхность можно представить как пересечение гиперповерхностей

$$
\begin{aligned}
& P_{1}=z_{1} x_{1}+z_{2} x_{2}, \\
& P_{2}=z_{1}\left(x_{2}^{3}+x_{3}^{3}-x_{4}^{3}\right)+z_{2}\left(x_{1}^{3}+x_{3}^{3}+x_{4}^{3}\right)
\end{aligned}
$$

в произведении пространств

$$
\begin{aligned}
& \mathbb{C} P_{1} \\
& \mathbb{C} P_{3}
\end{aligned}\left[\begin{array}{ll}
1 & 1 \\
3 & 3
\end{array}\right]
$$

В области $U_{11}=\left(\left\{z_{1} \neq 0\right\}\right) \cap\left(\left\{x_{1} \neq 0\right\}\right)$ с аффинными координатами $Z=z_{2} / z_{1}, X_{i}=$ $x_{i} / x_{1}, i=2,3,4$, это пересечение принимает вид

$$
\begin{gathered}
P_{1}=1+Z X_{2}=0 \\
P_{2}=X_{2}^{3}+X_{3}^{3}-X_{4}^{3}+Z\left(1+X_{3}^{3}+X_{4}^{3}\right)=0 \\
\left\{X_{2}, X_{3}\right\}=\frac{d X_{2} \wedge d X_{3} \wedge d P_{1} \wedge d P_{2}}{d X_{2} \wedge d X_{3} \wedge d X_{4} \wedge d Z}=-\frac{d X_{2} \wedge d X_{3}}{\operatorname{Jac}\left(\frac{P_{1}, P_{2}}{X_{4}, Z}\right)}=-3 X_{4}^{2}\left(X_{2}+1\right)
\end{gathered}
$$

Видно, что эти скобки неоднородны, что и следовало ожидать, поскольку ранее рассматривались взвешенные однородные координаты, а эти скобки имеют порядок 3 , как это и должно быть для "стандартной" поверхности $K 3$.

ЗАмечАниЕ 5.1. Подобно построениям в п. 4.2, общее определение принципа расшепления приводит к рациональным м неполиномиальным отображениям между произведениями кривых, поверхностей и т.д. в произведениях (взвешенных) проективных пространств. Поэтому на данный момент не ясно, как эта конструкция может быть связана с алгебрами $Q_{n, k}(\mathcal{E})$ и с их тензорными произведениями. Понимание смысла принципа расщепления на "квантовом" уровне может пролить свет на проблему "квантования" поверхности $K 3$ и ее слоений. 


\section{6. ИНТЕГРИРУЕМЫЕ СИСТЕМЫ}

В этом разделе приводятся различные примеры, когда исследуемые в данной статье полиномиальные пуассоновы структуры естественным образом появляются в подходе гамильтоновых систем. Понятие интегрируемости по Лиувиллю определено для алгебраических переменных неоднозначно, и его обсуждение выходит за рамки данной статьи (обсуждение этого вопроса см. в работе [26]). Здесь мы покажем лишь то, что простая конструкция аффинных скобок Пуассона иногда оказывается весьма полезной для прояснения свойств исходных гамильтоновых систем (обшее обсуждение данного вопроса см. в обзорной статье [27]).

6.1. Алгебры второго порядка, $n=4$. Рассмотрим пару квадрик общего положения в $\mathbb{C}^{4}$. Если

$$
p_{1}=\frac{1}{2}\left(x_{1}^{2}+x_{3}^{2}\right)+k x_{2} x_{4}, \quad p_{2}=\frac{1}{2}\left(x_{2}^{2}+x_{4}^{2}\right)+k x_{1} x_{3},
$$

то скобки принимают вид

$$
\begin{aligned}
& \left\{x_{i}, x_{i+1}\right\}=k^{2} x_{i} x_{i+1}-x_{i+2} x_{i+3}, \\
& \left\{x_{i}, x_{i+2}\right\}=k\left(x_{i+3}^{2}-x_{i+1}^{2}\right),
\end{aligned}
$$

где $i \equiv 0,1,2,3(\bmod 4)$. Квантование этих соотношений дает эллиптическую алгебру $\mathcal{Q}_{4}(\mathcal{E}, \eta)$, где $\mathcal{E} \subset \mathbb{C} P^{3}$ дается соотношениями $p_{1}=0, p_{2}=0$. Чтобы связать с этой пуассоновой структурой естественную интегрируемую систему, следует переписать эти два условия в “стандартной” форме Склянина $(3),(4)$ и выбрать в качестве гамильтониана переменную $x_{4}$. После этого из соотношений алгебры Склянина получается следующая гамильтонова система:

$$
\begin{aligned}
& \dot{x}_{1}=\left\{x_{1}, H\right\}=\left(J_{2}-J_{3}\right) x_{2} x_{3}, \\
& \dot{x}_{2}=\left\{x_{2}, H\right\}=\left(J_{1}-J_{3}\right) x_{1} x_{3}, \\
& \dot{x}_{3}=\left\{x_{3}, H\right\}=\left(J_{1}-J_{2}\right) x_{1} x_{2} .
\end{aligned}
$$

Это не что иное, как “волчкоподобное” представление (или представление эллиптического ротатора) классической двухчастичной эллиптической модели Руджинарса. Это наблюдение (сделанное на квантовом уровне Кричевером и Забродиным [28]) является предметом нашей статьи с Браденом и Горским [29].

ЗАМЕчАНИЕ 6.1. В данной схеме можно получить и обычный волчок Эйлера-Нама, если рассмотреть систему на коприсоединенных орбитах группы $S O(3, \mathbb{C})=S L_{2}(\mathbb{C})$, параметризованных значениями оператора Казимира $Q_{1}=1 / 2\left(x_{1}^{2}+x_{2}^{2}+x_{3}^{2}\right)=c_{1}$, и взять в качестве гамильтониана вторую квадрику $Q_{2}=1 / 2\left(J_{1} x_{1}^{2}+J_{2} x_{2}^{2}+J_{3} x_{3}^{2}\right)$. A именно,

$$
\begin{aligned}
& \dot{x}_{1}=\left\{x_{1}, H\right\}=J_{2} x_{2}\left\{x_{1}, x_{2}\right\}+J_{3} x_{3}\left\{x_{1}, x_{3}\right\}=\left(J_{3}-J_{2}\right) x_{2} x_{3}, \\
& \dot{x}_{2}=\left\{x_{2}, H\right\}=J_{1} x_{1}\left\{x_{2}, x_{1}\right\}+J_{3} x_{3}\left\{x_{2}, x_{3}\right\}=\left(J_{1}-J_{3}\right) x_{1} x_{3}, \\
& \dot{x}_{3}=\left\{x_{3}, H\right\}=J_{1} x_{1}\left\{x_{3}, x_{1}\right\}+J_{2} x_{2}\left\{x_{3}, x_{2}\right\}=\left(J_{2}-J_{1}\right) x_{1} x_{2},
\end{aligned}
$$

где была использована линейная регулярная пуассонова структура, ассоциированная с кривой $Q_{1}=1 / 2\left(x_{1}^{2}+x_{2}^{2}+x_{3}^{2}\right)=c_{1}$ в пространстве $\mathbb{C}^{3}:\left\{x_{i}, x_{j}\right\}=\epsilon_{i j k} \partial Q_{1} / \partial x_{k}=$ $\epsilon_{i j k} x_{k}$. 
6.2. Дваждыэллиптическая система. Глубокая связь между интегрируемыми системами и $N=2$ суперсимметричными калибровочными теориями была открыта Виттеном и Зайбергом (см. [30] и [31]). При этом известные классические многочастичные интегрируемые гамильтоновы модели (как непрерывные модели типа Калоджеро-Мозера, так и разностные модели Руджинарса) обнаружили новые неожиданные свойства, вытекающие из физических основ данных моделей. Описание шестимерных калибровочных теорий с присоединенными полями материи привело к появлению дваждыэллиптической системы, объединяюшей в единое семейство решения типа Калоджеро-Руджинарса и дуальные к ним и описьваемой гамильтонианом, содержащим эллиптическую зависимость как от координат, так и от импульсов [6], [7]. Покажем теперь, что $S U(2)$ дваждыэллиптическая система представляет собой пример $K 3$-подобной регулярной пуассоновой структуры, которая задает естественное гамильтоново описание данной системы.

Гамильтониан двухчастичной дваждыэллиптической системы в системе центра масс (в случае группы $S U(2))$ имеет следуюший вид [7]:

$$
H(p, q)=\alpha(q \mid k) \operatorname{cn}\left(p \beta(q \mid k, \tilde{k}) \mid \frac{\tilde{k} \alpha(q \mid k)}{\beta(q \mid k)}\right),
$$

где $\alpha(q \mid k)=\sqrt{1+g^{2} / \mathrm{sn}^{2}(q \mid k)}$, а $\beta(q \mid k, \tilde{k})=\sqrt{1+g^{2} \tilde{k} / \mathrm{sn}^{2}(q \mid k)}$ совпадает с $x_{5}$.

Выберем следуюшую систему четырех квадрик в $\mathbb{C}^{6}$, которая задает фазовое пространство двухчастичной дваждыэллиптической системы:

$$
\begin{aligned}
x_{1}^{2}-x_{2}^{2} & =1, \\
x_{1}^{2}-x_{3}^{2} & =k^{2}, \\
-g^{2} x_{1}^{2}+x_{4}^{2}-x_{5}^{2} & =1, \\
-g^{2} x_{1}^{2}+x_{4}^{2}+\tilde{k}^{-2} x_{6}^{2} & =\tilde{k}^{-2} .
\end{aligned}
$$

Первая пара уравнений этой системы задает “аффинизацию" проективного вложения эллиптической кривой в пространство $\mathbb{C} P^{3}$, в то время как вторая пара уравнений определяет эллиптическую кривую, локально расслоенную над первой эллиптической кривой. В случае, когда константа связи $g$ обрашается в нуль, полная система распадается в две копии эллиптической кривой, вложенные в $\mathbb{C} P^{3} \times \mathbb{C} P^{3}$. Подчеркнем, что ненулевая константа связи приводит к дополнительной некоммутативности между координатами в дополнение к стандартной некоммутативности между координатами и импульсами.

Следуя общей конструкции, получим скобки Пуассона для указанной системы квадрик:

$$
\begin{aligned}
& \left\{x_{1}, x_{2}\right\}=\left\{x_{1}, x_{3}\right\}=\left\{x_{2}, x_{3}\right\}=0, \\
& \left\{x_{5}, x_{1}\right\}=-x_{2} x_{3} x_{4} x_{6}, \\
& \left\{x_{5}, x_{2}\right\}=-x_{1} x_{3} x_{4} x_{6}, \\
& \left\{x_{5}, x_{3}\right\}=-x_{1} x_{2} x_{4} x_{6}, \\
& \left\{x_{5}, x_{4}\right\}=-g^{2} x_{1} x_{2} x_{3} x_{6}, \\
& \left\{x_{5}, x_{6}\right\}=0 .
\end{aligned}
$$


Заметим, что эта пуассонова структура сингулярна и не может быть расширена до голоморфной структуры на всем пространстве $\mathbb{C} P^{6}$ ввиду ограничения (6).

Из коммутационных соотношений следует, что $x_{6}$ - это константа, которая должна быть связана с постоянной энергией системы для обеспечения самосогласованности системы уравнений.

Ненулевой коммутатор между переменными координат двух торов отвечает стандартным скобкам Пуассона на фазовом пространстве, в то время как возможная нетривиальная скобка $\left\{x_{5}, x_{4}\right\}$ означала бы появление дополнительной некоммутативности в пространстве импульсов. Заметим, что тройка переменных $x_{1}, x_{2}, x_{3}$ в эллиптической параметризации принимает вид

$$
x_{1}=\frac{1}{\operatorname{sn}(q \mid k)}, \quad x_{2}=\frac{\operatorname{cn}(q \mid k)}{\operatorname{sn}(q \mid k)}, \quad x_{3}=\frac{\operatorname{dn}(q \mid k)}{\operatorname{sn}(q \mid k)},
$$

где функции Якоби sn, cn и dn отвечают “координатной” эллиптической кривой (тору с локальной координатой $q$ ).

ТЕОрема 6.1. Гамильтонова система с дваждығллиптическим гамильтонианом (20) әквивалентна следующей гамильтоновой системе, задаваемой скобками (21), с гамильтонианом $x_{5}$ :

$$
\begin{aligned}
& \dot{x}_{1}=x_{2} x_{3} x_{4} x_{6}, \\
& \dot{x}_{2}=x_{1} x_{3} x_{4} x_{6}, \\
& \dot{x}_{3}=x_{1} x_{2} x_{4} x_{6}, \\
& \dot{x}_{4}=g^{2} x_{1} x_{2} x_{3} x_{6}, \\
& \dot{x}_{6}=0 .
\end{aligned}
$$

ДокАЗАТЕЛЬСТво см. в работе [29].

В такой форме записи алгебраическая природа дваждыэллиптической системы видна особенно отчетливо, а сама система может быть явно проинтегрирована в терминах гиперэллиптических интегралов.

Можно дать и другое, полиномиальное описание данной системы, если заметить, что она имеет вид “элегантной” интегрируемой системы, обнаруженной Фэрли [32], для $n=4$ (при этом несушественная координата $x_{6}$ опускается):

$$
\begin{aligned}
& \dot{x}_{1}=x_{2} x_{3} x_{4}, \\
& \dot{x}_{2}=x_{1} x_{3} x_{4}, \\
& \dot{x}_{3}=x_{1} x_{2} x_{4}, \\
& \dot{x}_{4}=g^{2} x_{1} x_{2} x_{3} .
\end{aligned}
$$

Эта система допускает красивое представление, в котором она имеет вид расцепленной пары волчков Эйлера-Нама после следуюшей замены переменных:

$$
\begin{array}{lll}
u_{+}=x_{3} x_{4}+g^{2} x_{1} x_{2}, & v_{+}=x_{2} x_{4}+g^{2} x_{1} x_{3}, & w_{+}=x_{1} x_{4}+g^{2} x_{3} x_{2} \\
u_{-}=x_{3} x_{4}-g^{2} x_{1} x_{2}, & v_{-}=x_{2} x_{4}-g^{2} x_{1} x_{3}, & w_{-}=x_{1} x_{4}-g^{2} x_{3} x_{2} .
\end{array}
$$


В этих новых переменных дваждыэллиптическая система (22) становится эквивалентной двум расцепленным системам

$$
\dot{u}_{+}=v_{+} w_{+}, \quad \dot{v}_{+}=w_{+} u_{+}, \quad \dot{w}_{+}=u_{+} v_{+}
$$

и

$$
\dot{u}_{-}=v_{-} w_{-}, \quad \dot{v}_{-}=w_{-} u_{-}, \quad \dot{w}_{-}=u_{-} v_{-} .
$$

Геометрически эта замена переменных отвечает переходу к прямому (расцепленному) произведению $\mathcal{E}_{+} \times \mathcal{E}_{-}$двух эллиптических кривых, заданных соответствующими операторами Казимира моделей (23) и (24):

$$
\begin{aligned}
\mathcal{E}_{+}: & u_{+}^{2}-v_{+}^{2}=k^{2}\left(E^{2}-1\right), \\
u_{+}^{2}-w_{+}^{2} & =\left(k^{2}-2\right)\left(E^{2}-1\right)
\end{aligned}
$$

$$
\begin{aligned}
\mathcal{E}_{-}: & u_{-}^{2}-v_{-}^{2}=k^{2}\left(E^{2}-1\right), \\
u_{-}^{2}-w_{-}^{2} & =\left(k^{2}-2\right)\left(E^{2}-1\right) .
\end{aligned}
$$

Этот результат напоминает теорему Уорда [33], которая утверждает, что дифференциальный оператор второго порядка с потенциалом Ламэ $n(n+1) /\left(2 \mathrm{sn}^{2}(q \mid k)\right)$ может быть разложен в произведение двух дифференциальных матричных операторов первого порядка $\partial+A$ и $\partial-A$, где матрично-значные функции $A=\left(A_{1}, A_{2}, A_{3}\right)$ удовлетворяют системе уравнений Нама:

$$
\dot{A}_{i}=\epsilon_{i j k}\left[A_{j}, A_{k}\right] .
$$

\section{3. Дваждыэллиптическая система как пример системы Намбу-Гамиль-}

тона. Полиномиальная пуассонова структура, описывающая дваждыэллиптическую систему, также представляет собой пример структуры Намбу-Пуассона (см. п. 2.1).

Конструкции, описываемые с помощью квадрик, дают нетривиальные примеры динамических систем Намбу-Гамильтона. Именно, система из трех квадрик в пространстве $\mathbb{C}^{5}$,

$$
\begin{aligned}
x_{1}^{2}-x_{2}^{2} & =1, \\
x_{1}^{2}-x_{3}^{2} & =k^{2}, \\
-g^{2} x_{1}^{2}+x_{4}^{2}+x_{5}^{2} & =1,
\end{aligned}
$$

имеет сечение, задаваемое условием $x_{5}=E$, в то время как однопараметрическое пересечение трех квадрик в пространстве $\mathbb{C}^{4}$,

$$
\begin{aligned}
& Q_{1}=x_{1}^{2}-x_{2}^{2}=1, \\
& Q_{2}=x_{1}^{2}-x_{3}^{2}=k^{2}, \\
& Q_{3}=-g^{2} x_{1}^{2}+x_{4}^{2}=1-E^{2} .
\end{aligned}
$$

определяет систему Намбу-Гамильтона (которая есть не что иное, как обсуждавшаяся выше дваждыэллиптическая система!)

$$
\frac{d x_{i}}{d t}=\left\{Q_{1}, Q_{2}, Q_{3}, x_{i}\right\}
$$


Благодарности. Авторы благодарят Г. Брадена и А. Горского за сотрудничество и плодотворные обсуждения. Авторы особенно благодарны А. Морозову, интерес которого к данной деятельности был для них особенно воодушевляющим. В.Р. благодарен А. Маршакову, А. Миронову, Н. Некрасову и А. Турбинеру, многочисленные обсуждения с которыми были весьма полезны. Оба автора выражают свою признательность Б. Энрикесу за дружеское участие и полезные обсуждения. А.О. благодарит Университет г. Анжер, где была написана основная часть текста, за стимулирующую рабочую атмосферу. В.Р. благодарен Б. Хесину за плодотворные обсуждения и теплое гостеприимство во время пребывания в г. Торонто. Мы признательны Л. Чехову, чьи многочисленные предложения и замечания улучшили текст статьи.

Работа А.О. была частично поддержана Российским фондом фундаментальных исследований (гранты № 99-01-01669, 00-15-96579), CRDF (грант № RP1-2254) и INTAS (грант № 00-00055). Работа В.Р. была частично поддержана Российским фондом фундаментальных исследований (гранты № 01-01-00549, 00-15-96557) и INTAS (грант № 99-1705).

\section{Список литературы}

[1] Б. Фейгин, А. Одесский. Функц. анализ и его прилож. 1989. Т. 23. № 3. С. 45-54.

[2] B. Feigin, A. Odesskii. Vector bundles on an elliptic curve and Sklyanin algebras. In: Topics in quantum groups and finite-type invariants, 65-84. Amer. Math. Soc. Transl. Ser. 2, 185. Providence, RI: Amer. Math. Soc., 1998.

[3] I. Vaisman. Lectures on the Geometry of Poisson Manifolds. Progress in Mathematics. V. 118. Basel: Birkhäuser, 1994.

[4] A. Weinstein. J. Diff. Geom. 1983. V. 18. № 3. P. 523-557.

[5] E. K. Склянин. Функц. анализ и его прилож. 1982. Т. 16. № 4. С. 27-34.

[6] V. Fock, A. Gorsky, N. Nekrasov, V. Rubtsov. Duality in integrable systems and gauge theories J. High Energy Phys. 2000. № 7. Paper 28.

[7] H. W. Braden, A. Marshakov, A. Mironov, A. Morozov. Nucl. Phys. B. 2000. V. 573. № 1-2. P. 553-572; hep-th/9906240.

[8] J. T. Stafford, M. Van den Bergh. Bull. Am. Math. Soc. 2001. V. 38. № 2. P. 171-216.

[9] H. Kim, C.-T. Lee. Phys. Lett. B. 2002. V. 536. P. 154-160; hep-th/0105265.

[10] P. Candelas, A. M. Dale, C. A. Lutken, R. Schimmrigk. Nucl. Phys. B. 1988. V. 298. № 3. P. 493-525.

[11] P. Candelas, de la Ossa, Xenia C., P. S. Green, L. Parkes. Nucl. Phys. B. 1991. V. 359. № 1. P. 21-74.

[12] Y. Nambu. Phys. Rev. D. Ser. 3. 1973. V. 7. P. 2405-2412.

[13] L. Takhtajan. Commun. Math. Phys. 1994. V. 160. № 2. P. 295-315.

[14] J. A. de Azcárraga, A. M. Perelomov, J. C. Pérez Bueno. J. Phys. A. 1996. V. 29. № 24. P. 7993-8009.

[15] G. Khimshiashvili. Proc. A. Razmadze Math. Inst. 1999. V. 119. P. 111-120.

[16] B. Dubrovin. Differential geometry of the space of orbits of a Coxeter group. In: Surveys in Differential Geometry. V. IV. Ed. Chun-Lian Terng. Cambridge, MA: Int. Press, 1998. P. 181-211.

[17] M. Ugaglia. Int. Math. Res. Notices. 1999. № 9. P. 473-493.

[18] Ph. Boalch. Invent. Math. 2001. V. 146. P. 479-506; math.DG/0011062.

[19] L. O. Chekhov, V. V. Fock. Czech. J. Phys. 2000. V. 50. № 11. P. 1201-1208. 
[20] J. E. Nelson, T. Regge. Phys. Lett. B. 1991. V. 272. P. 213-216; Commun. Math. Phys. 1993. V. 155. P. 561-568.

[21] A. Bondal. A symplectic groupoid of triangular bilinear forms and the braid group. Preprint IHES M2000/02.

[22] A. Korovnichenko, A. Zhedanov. Dual algebras with non-linear Poisson brackets. In: Integrable Structures of Exactly Solvable Two-Dimensional Models of Quantum Field Theory. NATO Science series. V. 35. Eds. S. Pakuliak, G. von Gehlen. Dordrecht: Kluwer, 2001. P. 265-272.

[23] S. Mukai. (Japanese) Sugaku Expositions. 1988. V. 1. № 2. P. 139-174; Sugaku. 1987. V. 39. № 3. P. 216-235.

[24] E. Cattani, D. Cox, A. Dickenstein. Compos. Math. 1997. V. 108. № 1. P. 35-76.

[25] M. Lynker, R. Schimmrigk. Nucl. Phys. B. 1997. V. 484. № 3. P. 562-582.

[26] P. Vanhaecke. Integrable Systems in the Realm of Algebraic Geometry. Lecture Notes in Mathematics. V. 1638. Berlin: Springer, 1996.

[27] M. Adler, P. van Moerbeke. Commun. Math. Phys. 1982. V. 83. № 1. P. 83-106.

[28] И. Кричевер, А. Забродин. УМН. 1995. Т. 50. № 6. С. 3-56.

[29] H. Braden, A. Gorsky, A. Odesskii, V. Rubtsov. Nucl. Phys. B. 2002. V. 633. P. 414-442; hep-th/0111066.

[30] A. Gorsky, I. Krichever, A. Marshakov, A. Mironov, A. Morozov. Phys. Lett. B. 1995. V. 355. P. 466-474.

[31] R. Donagi, E. Witten. Nucl. Phys. B. 1996. V. 460. № 2. P. 299-334.

[32] D. B. Fairlie. Phys. Lett. A. 1987. V. 119. № 9. P. 438-440.

[33] R. S. Ward. J. Phys. A. 1987. V. 20. № 10. P. 2679-2683.

Поступила в редакцию 14.XII.2001 г. 